\title{
The role of helium droplets in the mass spectra of diatomics: the suppression of dissociative reactions
}

\author{
Andrew M. Ellis and Shengfu Yang* \\ The Nanochemistry Group, Department of Chemistry, University of Leicester \\ University Road, Leicester, LE1 7RH, UK
}

* Corresponding authors. Email addresses: sfy1@le.ac.uk.

Tel: +44 (0)116 252 2127; Fax: +44 (0)116 2523789.

Manuscript submitted to Chinese Journal of Physical Chemistry 


\begin{abstract}
It is generally accepted that electron impact of doped helium nanodroplets initially produces a positively charged helium atom, which then ionizes the dopant if the two come into contact. In effect the $\mathrm{He}^{+}$can initiate ion-molecule reactions. However, the effect of the surrounding helium on ion-molecule reactions remains ambiguous. To explore this, electron-induced chemistry has been investigated for the diatomic molecules $\mathrm{O}_{2}, \mathrm{CO}$ and $\mathrm{N}_{2}$. The helium is found to significantly suppress dissociative ion product channels.
\end{abstract}




\section{Introduction}

Molecules embedded in helium nanodroplets are commonly investigated by optical spectroscopy and/or mass spectrometry. When electron ionization is employed in mass spectrometry molecules in the interior of helium nanodroplets are ionized indirectly. The ionization process starts with the formation of $\mathrm{He}^{+}$somewhere within the droplet, which then undergoes a resonant hopping mechanism. ${ }^{1}$ This positive hole can hop from atom to atom about 10 times before localizing, yielding helium cluster ions. ${ }^{2,3}$ After encountering the dopants, $\mathrm{He}^{+}$can transfer the charge to the dopants, which is always energetically favourable because the ionization energy of any molecular dopant is well below that of helium. The reactions between $\mathrm{He}^{+}$and the neutral dopants can lead to molecular ions being detected in the gas phase by mass spectrometry, although precisely how such ions leave the droplet is not yet established. An alternative means of charge transfer starts from $\mathrm{He}^{-}$, which can act as a two-electron donor and produce both anions and cations. ${ }^{4}$ When an electron strikes a helium droplet the production of metastable electronically excited helium $\left(\mathrm{He}^{*}\right)$ is another alternative to ionization, and therefore ionization of dopant molecules is also possible by Penning ionization. ${ }^{5,6}$ Ionization through $\mathrm{He}^{-}$and $\mathrm{He}^{*}$ generally occurs in larger helium droplets composed of $>10^{5}$ helium atoms.

Helium nanodroplets are liquid droplets with a steady-state temperature of $0.38 \mathrm{~K}$ for ${ }^{4} \mathrm{He} .{ }^{7}$ This low temperature, the potentially very high cooling rate provided by the evaporative loss of helium atoms, and the very high thermal conductivity of superfluid helium, makes this an interesting environment in which to study ion-molecule chemistry. Helium droplets can, in principle, reduce the fragmentation of molecular ions through rapid quenching and can therefore aid soft ionization. However, examples studied so far indicate that ion fragmentation is only marginally reduced by the helium when compared with gas-phase electron-induced mass spectra. ${ }^{5,8-11}$ Exceptions include cyclopentanol, cyclohexanol ${ }^{10}$ and triphenylmethanol, ${ }^{2}$ which all show significant reductions in ion fragmentation.

The role of helium nanodroplets in ion-molecule reactions remains unclear. In a photoionization experiment, Braun and Drabbels used UV light at $266 \mathrm{~nm}$ to ionize a $\mathrm{CF}_{3} \mathrm{I}$ molecule embedded in the helium nanodroplet. ${ }^{12}$ They found that the neutral $\mathrm{CF}_{3}$ 
fragment escapes via a direct mechanism, and only partially transfers its excess kinetic energy to helium nanodroplets. Fárník and Toennies investigated the ionization of binary molecular clusters of $\mathrm{N}_{2} / \mathrm{D}_{2}$ and $\mathrm{CH}_{4} / \mathrm{D}_{2}$ and found that ionization of molecular clusters in helium droplets starts with charge transfer reactions between $\mathrm{He}^{+}$and one of the dopant molecules, followed by secondary reactions between the primary charge transfer products and the remaining neutral molecules. ${ }^{13}$ They also showed that ion fragmentation dynamics can be interpreted by reaction rates at the gas phase reactions and the reaction enthalpy.

Using a sequential pickup method, work in our laboratory has investigated ionmolecule reactions between water clusters and a co-dopant in a core-shell structure, with small water clusters as the core. ${ }^{14}$ Reduction in the fragmentation of water clusters was clearly observed by adding a shell of non-polar molecules around the water clusters, such as $\mathrm{N}_{2}, \mathrm{O}_{2}, \mathrm{CO}_{2}$ and $\mathrm{C}_{6} \mathrm{D}_{6}$. However, this softening effect was accounted by an "energy-in and energy-out" mechanism within the "hot" clusters following the primary charge transfer reactions between $\mathrm{He}^{+}$and the non-polar shell molecules, rather than cooling by the ultra-cold helium nanodroplets. More recently, Leidlmair et al. investigated the ionization of large methane clusters in helium nanodroplets and found a striking similarity between the mass spectra for gas-phase and helium nanodroplet studies, ${ }^{15}$ suggesting that helium nanodroplets have a negligible effect on the subsequent ionmolecule chemistry after the positive charge is transferred to the methane clusters.

In this work, we explore the role of helium nanodroplets in primary charge transfer reactions activated by electron impact. To simplify the investigation we have chosen several diatomic molecules, namely, $\mathrm{N}_{2}, \mathrm{O}_{2}$ and $\mathrm{CO}$, because they have very limited ion fragmentation channels when reacting with $\mathrm{He}^{+}$. We investigate electron impact mass spectrometry with only one type of molecule added to helium nanodroplets, from which primary reaction products can be identified and the influence of helium on the primary reaction can then be established by comparison with gas phase mass spectra.

\section{Experimental}


The experiments were carried out using a pulsed helium nanodroplet instrument, which has been described in detail previously. ${ }^{16-18}$ Briefly, a pulsed valve (General Valve series 99) is attached to a home-made face plate, with the seal between the two being made by indium wire acting as a gasket. The poppet is made from Kel-F and the faceplate has a $0.5 \mathrm{~mm}$ aperture through which expansion occurs. At a stagnation pressure of $20 \mathrm{bar}$ and a source temperature of $15 \mathrm{~K}$, the average helium nanodroplet size is $\langle N\rangle \approx 7500$ and these conditions were chosen for the experiments reported here. ${ }^{16}$ The droplet beam was skimmed and then entered a pick-up cell with an inner diameter of $6 \mathrm{~mm}$. The pickup chamber and the time-of-flight mass spectrometer (TOF-MS) are connected via a $4 \mathrm{~mm}$ diameter aperture in order to reduce the free diffusion of gas samples into the mass spectrometer. The partial pressure in the TOF-MS is typically $10^{-8}$ mbar, which eliminates the possibility of reactions caused by collisions between ion products and background gases. The partial pressure of gas samples in the pickup cell was controlled via a needle valve, allowing one molecule to be added to the droplets on average. As detailed later, $\mathrm{O}_{2}^{+}, \mathrm{N}_{2}^{+}$and $\mathrm{CO}^{+}$were prominent ion signals in the mass spectra although large cluster ions were also seen.

\section{Results and Discussion}

Figure 1 shows the mass spectra of $\mathrm{O}_{2}$ clusters. The relative abundance of $\mathrm{O}^{+}$is hard to determine from the mass spectrum because it overlaps with $\mathrm{He}_{4}{ }^{+}$at $\mathrm{m} / \mathrm{z} 16$ channel. The only significant odd-numbered $\mathrm{O}_{n}^{+}$cluster ion in the mass spectrum is $\mathrm{O}_{5}^{+}$, which has an abundance equal to $\sim 10 \%$ of the overall even-numbered $\mathrm{O}_{n}^{+}$ions.

The mass spectrum derived from the addition of $\mathrm{CO}$ is shown in Figure 2. The $\mathrm{C}^{+}$ and $\mathrm{O}^{+}$ions, will overlap with $\mathrm{He}_{3}{ }^{+}$and $\mathrm{He}_{4}{ }_{4}$ at $\mathrm{m} / z, 12$ and $\mathrm{m} / z, 16$, respectively, making it difficult to determine their abundances. For ${ }^{12} \mathrm{C}^{+}$ion product we refer to our previous work on ${ }^{13} \mathrm{CO}$, which has shown less than $1 \% \mathrm{C}^{+}$abundance relative to $\mathrm{CO}^{+}{ }^{18}$ For the $\mathrm{O}^{+}$ ion, even if it were produced, it should have very low abundance in a similar way to the $\mathrm{O}^{+}$product in $\mathrm{O}_{2}$ clusters, because the $m / z 16$ ion signal barely has any difference from the $\mathrm{He}_{4}{ }^{+}$ion in the mass spectrum of helium droplets without the addition of diatomics. For $\mathrm{N}_{2}$, the $\mathrm{N}^{+}$signal observed in the mass spectra is about $3 \%$ of that of $\mathrm{N}_{2}{ }^{+}$, which is produced partly from the gas phase $\mathrm{N}_{2}$ in the vacuum chamber. In the gas phase mass 
spectrum $\mathrm{N}^{+}$has an abundance of $15 \% ;{ }^{19}$ hence the residual $\mathrm{N}_{2}$ in the vacuum chamber can increase the overall $\mathrm{N}^{+}$product, and the $\mathrm{N}^{+}$ion generated by dissociation of $\mathrm{N}_{2}$ during the charge transfer reaction should be even lower. The only odd-number nitrogen cluster ion observed is $\mathrm{N}_{3}^{+}$(see Figure 3), which has an abundance less than $1 \%$ of $\mathrm{N}_{2}^{+}$.

To understand the role of helium nanodroplets in the charge transfer reactions we first look at the reaction pathways in the gas phase between $\mathrm{He}^{+}$and the selected diatomics, which are strongly in favour of the favour dissociative products. At room temperature, the $\mathrm{He}^{+}+\mathrm{N}_{2}$ reaction has a branching ratio of $60 \%$ for the dissociative channel leading to $\mathrm{N}^{+}+\mathrm{N} ;{ }^{20}$ while $\mathrm{C}^{+}$is the dominant reaction product for $\mathrm{He}^{+}+\mathrm{CO}$ in the gas phase. ${ }^{21}$ For $\mathrm{O}_{2}$ the reactions with $\mathrm{He}^{+}$give rise to $\mathrm{O}^{+}$with a yield as high as $97 \% .^{20,22}$

We interpret the marked difference between the gas phase and helium droplet mass spectra in terms of reaction energetics and explore the role of superfluid helium droplets in the reactions, i.e., either the dissociative reactions are suppressed by the droplets, or helium droplets act as a reservoir that allows the dissociative reaction products to recombine into molecular ions. We will focus on the energy released during the charge reactions, which can be dissipated through evaporative loss of helium atoms if the dissociative reactions occur as in the gas phase. Otherwise the dissociative ion products would be ejected from the droplets, resulting in ion abundance similar to that in the gas phase mass spectra.

Using the NIST database, ${ }^{19}$ the energetics for dissociative charge transfer reactions can be calculated. For $\mathrm{N}_{2}$, the dissociative reaction, $\mathrm{He}^{+}+\mathrm{N}_{2} \rightarrow \mathrm{He}+\mathrm{N}+\mathrm{N}^{+}$, will only release $0.26 \mathrm{eV}$ energy, which, in principle, can be dissipated by evaporation of $\sim 400$ helium atoms from the helium nanodroplets (each removing $5 \mathrm{~cm}^{-1}$ energy ${ }^{7}$ ). The average helium nanodroplet size is $\langle N\rangle=7500$ in our work; so the droplets can easily accommodate the required loss of helium atoms. As a result no helium droplets will be destroyed by dissipation of the released energy, and $\mathrm{N}^{+}$and $\mathrm{N}$ can remain inside helium nanodroplets if they are generated in the charge transfer reaction. Subsequently $\mathrm{N}^{+}$and $\mathrm{N}$ can recombine into $\mathrm{N}_{2}^{+}$in the droplets.

For $\mathrm{CO}$, the $\mathrm{He}^{+}+\mathrm{CO} \rightarrow \mathrm{He}+\mathrm{C}^{+}+\mathrm{O}(\Delta E=-2.17 \mathrm{eV})$ reaction releases more energy than $\mathrm{He}^{+}+\mathrm{N}_{2}$, which could result in the evaporation of $\sim 3500$ helium atoms. This 
means that for helium nanodroplets containing less than 3500 helium atoms (corresponding to $20 \%$ of the total helium nanodroplets assuming a log-normal distribution $^{7}$ ), the dissociative reaction energy cannot be dissipated through evaporation of helium atoms. As a result the $\mathrm{C}^{+}$and $\mathrm{O}$ atoms produced through this route in small droplets would be ejected, resulting in a mass spectrum with significant abundance of $\mathrm{C}^{+}$ ions. In our experiments a very low yield of $\mathrm{C}^{+}$ions is observed in the mass spectrum $(<$ $1 \%$ ), suggesting that the dissociative reaction channel has been suppressed in the charge transfer reaction between $\mathrm{He}^{+}$and $\mathrm{CO}$.

Even stronger evidence for the suppression of dissociative channels in the charge transfer reactions between $\mathrm{He}^{+}$and diatomic molecules has been provided in the case of $\mathrm{O}_{2}$. The reaction $\mathrm{He}^{+}+\mathrm{O}_{2} \rightarrow \mathrm{He}+\mathrm{O}^{+}+\mathrm{O}(\Delta E=-5.80 \mathrm{eV})$ is more exothermic, which is able to remove $\sim 9000$ helium atoms. In this case about $75 \%$ helium nanodroplets cannot retain $\mathrm{O}^{+}$and $\mathrm{O}$ through evaporative cooling. If the $\mathrm{He}^{+}+\mathrm{O}_{2}$ reaction in helium droplets has a similar branching ratio for production of $\mathrm{O}^{+}$as in the gas phase mass spectra (97\%), odd-numbered oxygen cluster ions would be prominent in the mass spectrum. An abundance of $\sim 10 \%$ for the $\mathrm{O}_{5}{ }^{+}$ion in the mass spectrum strongly suggests that helium droplets have suppressed the dissociative reaction channel.

Finally, we discuss the influence of clusters to the fragmentation of molecules

during the charge transfer reactions. For $\mathrm{CO}$, our previous experiments using ${ }^{13} \mathrm{CO}$ has shown negligible abundance of ${ }^{13} \mathrm{C}^{+}$, and its dominant product is $\mathrm{CO}^{+} .{ }^{18}$ For $\mathrm{N}_{2}$ and $\mathrm{O}_{2}$, the binding energies in clusters are known to be no more than $200 \mathrm{~cm}^{-1}$, meaning that the influence of the clusters should be negligible to the fragmentation patterns.

\section{Conclusions}

In this work we investigate the ionization of diatomic clusters in superfluid helium nanodroplets in order to identify the roles of helium droplets. For $\mathrm{O}_{2}, \mathrm{CO}$ and $\mathrm{N}_{2}$ we have obtained mass spectra dominated by molecular cluster ions, which is drastically different from the gas phase reactions between $\mathrm{He}^{+}$and the selected diatomics. This suggests that helium nanodroplets either suppress the dissociative reaction channel or serve as an environment that allows the dissociative reaction products to recombine. To interpret the 
results and understand the role of helium droplets we have calculated the reaction enthalpies assuming that dissociative reactions do occur, which would have to be dissipated by the helium droplets. For $\mathrm{N}_{2}$ the dissociative $\mathrm{He}^{+}+\mathrm{N}_{2}$ reaction releases a low energy of $0.26 \mathrm{eV}$ only, which is not indicative for the role of helium droplets. For $\mathrm{CO}$ and $\mathrm{O}_{2}$, we have found that the reaction enthalpies are too high to be dissipated by evaporation of helium atoms, in particular, in relatively small helium droplets. Hence the dissociative reaction channels of diatomics must have been suppressed by helium nanodroplets.

\section{Acknowledgements}

SFY and AME wish to thank the UK EPSRC and the Leverhulme Trust for grants in support of this work.

\section{References}

1. K. R. Atkins, in Proceedings of the International School of Physics "Enrico Fermi," Course XXI on Liquid Helium, edited by G. Carerei, Academic, New York, 1963, p. 403.

2. W. K. Lewis, B. E. Applegate, J. Sztáray, B. Sztáray, T. Baer, R. J. Bemish and R. E. Miller, J.Am. Chem. Soc. 126 (2004) 11283.

3. A. M. Ellis and S. Yang, Phys. Rev. A 76 (2007) 032714.

4. A. Mauracher, M. Daxner, S. E. Huber, J. Postler, M. Renzler, S. Denifl, P. Scheier and A. M. Ellis, J. Chem. Phys. 142 (2015) 104306.

5. M. Lewerenz, B. Schilling and J. P. Toennies, J. Chem. Phys. 102 (1995) 8191.

6. A. A. Scheidemann, V. V. Kresin and H. Hess, J. Chem. Phys. 107 (1997) 2839.

7. J. P. Toennies and A. F. Vilesov, Angew. Chemie Int. Ed. 43 (2004) 2622.

8. A. Sheidemann, B. Schilling and J. P. Toennies, J. Phys. Chem. 97 (1993) 2128.

9. S. Yang, S. M. Brereton, M. D. Wheeler and A. M. Ellis, J. Phys. Chem. A 110 (2006) 1791.

10. S. Yang, S. M. Brereton, M. D. Wheeler and A. M. Ellis, Phys. Chem. Chem. Phys. 
7 (2005) 4082.

11. S. Yang, S. M. Brereton and A.M. Ellis, Intl. J. Mass. Spectrom. 253 (2006) 79.

12. A. Braun and M. Drabbels, Phys. Rev. Lett. 93 (2004) 253401.

13. M. Fárník and J. P. Toennies, J. Chem. Phys. 122 (2005) 014307.

14. J. Liu, B. Shepperson, S. Yang and A. M. Ellis, Phys. Chem. Chem. Phys. 13 (2011) 13920.

15. C. Leidlmair, P. Bartl, H. Schöbel, S. Denifl, T. D. Märk, S. Yang, A. M. Ellis and P. Scheier, ChemPhysChem 13 (2012) 469.

16. S. Yang, S. M. Brereton and A. M. Ellis, Rev. Sci. Instrum. 76 (2005) 104102.

17. S. Yang and A. M. Ellis, Rev. Sci. Instrum. 79 (2008) 016106.

18. B. Shepperson, J. Liu, A. M. Ellis and S. Yang, J. Phys. Chem. A 115 (2011) 7010.

19. The NIST Chemistry WebBook, http://webbook.nist.gov/chemistry/.

20. N. G. Adams and D. Smith, J. Phys. B: Atom. Molec. Phys. 9 (1976) 1439.

21. L. B. Zhao, P. C. Stancil, J. P. Gu, H.-P. Liebermann, Y. Li, P. Funke, R. J. Buenker, B. Zygelman, M. Kimura and A. Dalgarno, Astrophys. J. 615 (2004) 1063.

22. R. Johnsen, J. A. MacDonald and M.A. Biondi, J. Chem. Phys. 67 (1977) 4718. 


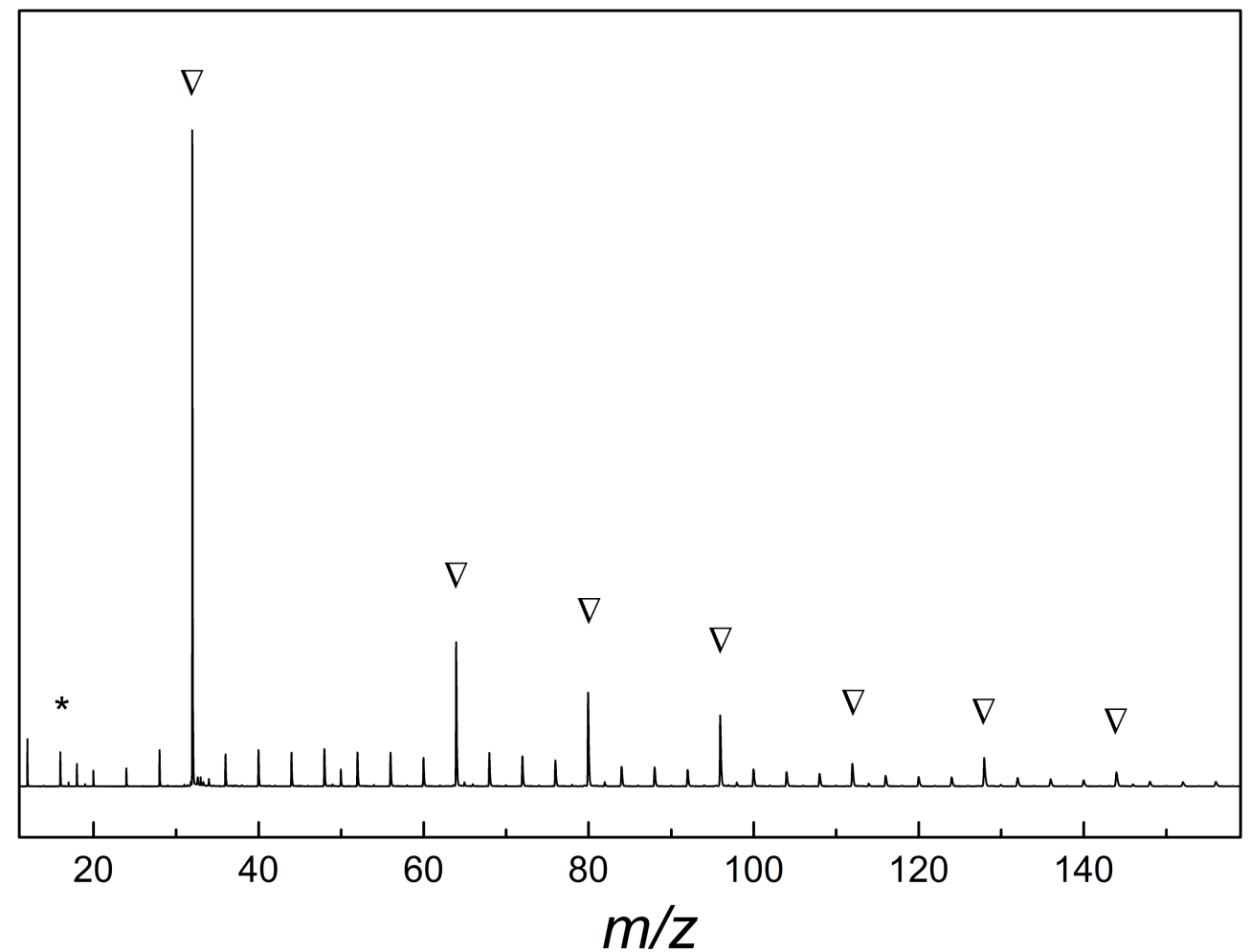

Figure 1. Mass spectrum of $\mathrm{O}_{2}$ clusters in helium nanodroplets. The asterisk marks the $m / z 16$ peak, and the triangles mark $\mathrm{O}_{n}{ }^{+}$ions $(n=2,4-9)$. 


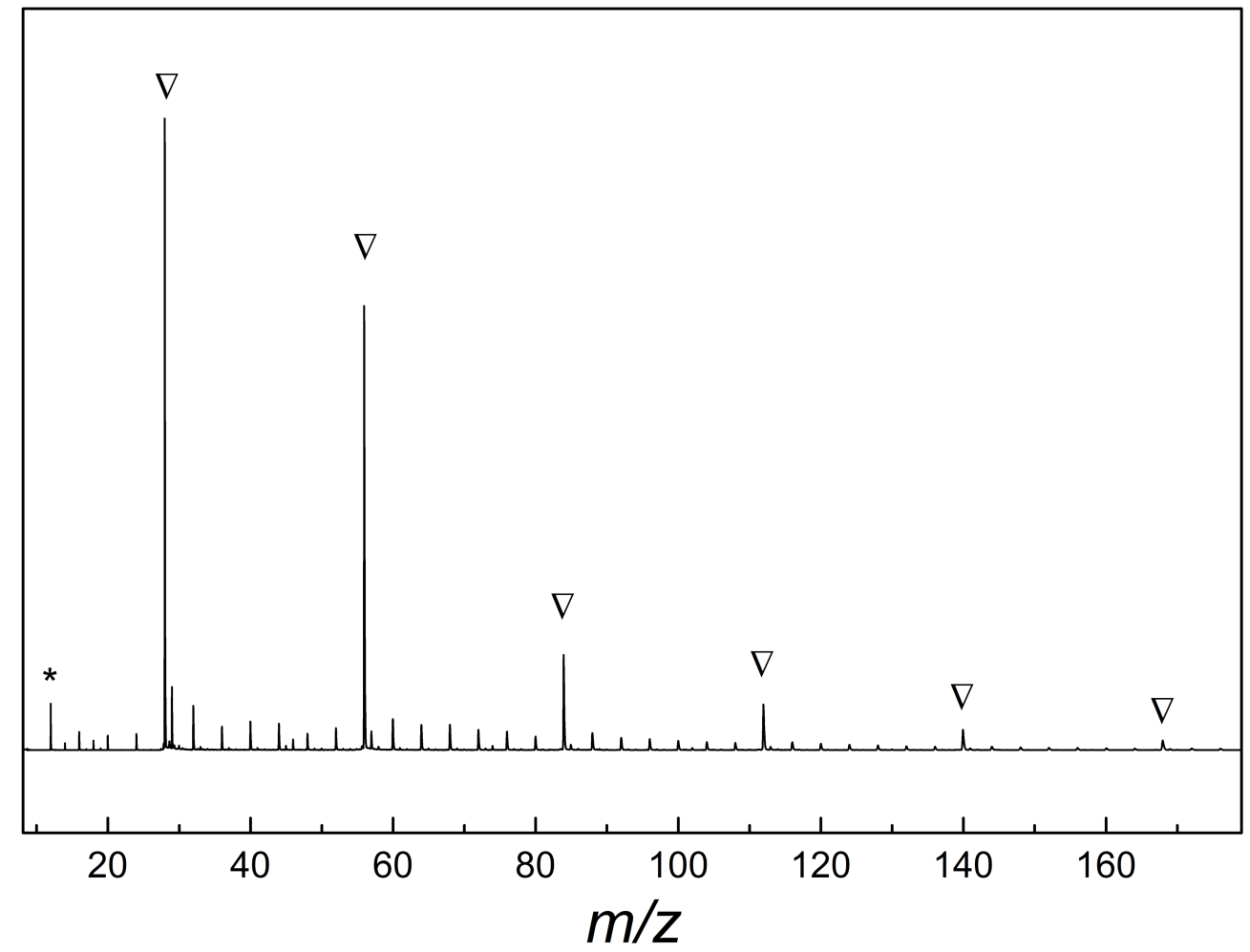

Figure 2. Mass spectrum of $\mathrm{CO}$ clusters in helium nanodroplets. The asterisk indicates the $m / z, 12$ peak, and the triangles mark $\mathrm{CO}_{n}{ }^{+}$ions $(n=1-6)$. 


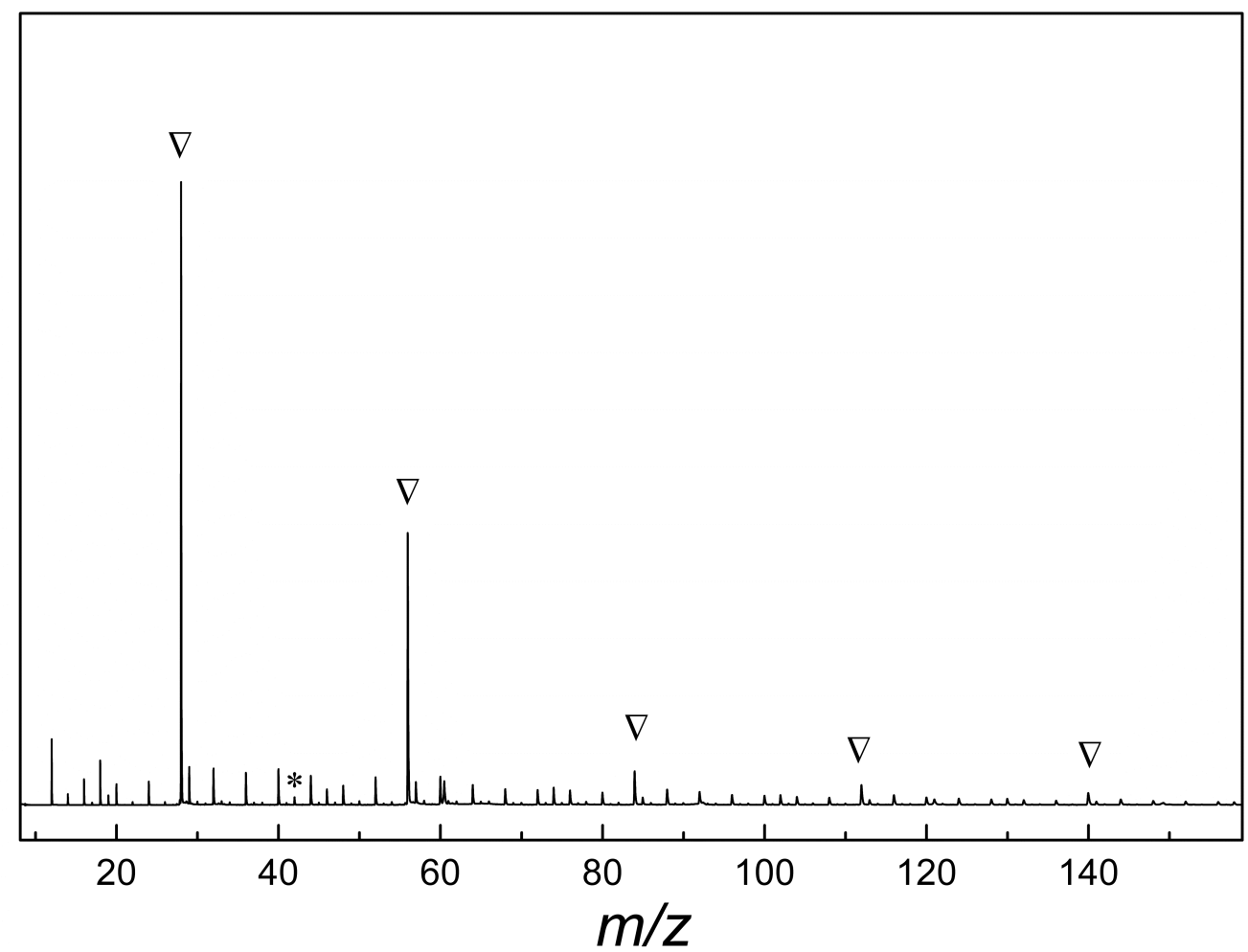

Figure 3. Mass spectrum of $\mathrm{N}_{2}$ clusters in helium nanodroplets. The asterisk indicates the $m / z 42$ peak, and the triangles mark $\mathrm{N}_{2 n}{ }^{+}$ions $(n=1-5)$. 